\title{
A Diminished Role for the Sarcoplasmic Reticulum in Newborn Myocardial Contraction: Effects of Ryanodine
}

\author{
THOMAS S. KLITZNER AND WILLIAM F. FRIEDMAN \\ The Division of Pediatric Cardiology, Department of Pediatrics, University of California at Los Angeles School of \\ Medicine and UCLA Medical Center, Los Angeles, California 90024
}

\begin{abstract}
Ryanodine, known to reduce $\mathrm{Ca}^{2+}$ release from the sarcoplasmic reticulum (SR), was used in conjunction with the single sucrose gap voltage clamp technique to study excitation-contraction coupling in papillary muscles isolated from newborn and adult rabbits. Ryanodine altered the components of voltage clamp-induced tension in the adult producing a pattern that closely resembled the normal newborn. The phasic component of tension in mature myocardium was markedly and significantly reduced by ryanodine. In addition, ryanodine significantly altered the voltage dependence of this component of tension, suggesting a change in its mechanism of generation. The appearance of a prominent phasic component of tension, absent normally, but produced in the newborn by $\mathrm{Ca}^{2+}$ loading, was also abolished by ryanodine. These results support previous proposals that the phasic component of voltage clamp-induced tension in mammalian myocardium is produced by the release of $\mathrm{Ca}^{2+}$ from the SR. In the newborn, ryanodine caused a significantly smaller decrease in the ratio of phasic to tonic tension than in the adult, suggesting a much less significant role for the $S R$ in excitation-contraction coupling in the younger age group. Although ryanodine had a significant effect on phasic tension, no alteration in the amplitude or voltage dependence of tonic tension, generated by transmembrane $\mathrm{Ca}^{2+}$ influx, could be demonstrated in either newborn or adult heart. This investigation suggests that in newborn myocardium, $\mathrm{Ca}^{2+}$ release from the $\mathrm{SR}$ plays a negligible role in excitation-contraction coupling, which depends, rather, on the influx of $\mathrm{Ca}^{2+}$ from the extracellular space. (Pediatr Res 26: 98-101, 1989)
\end{abstract}

\section{Abbreviations}

$P / T$, ratio of phasic tension to tonic tension $\mathrm{SR}$, sarcoplasmic reticulum

It has recently been demonstrated that immature mammalian ventricular tissue responds to voltage clamp depolarization by developing tension which increases monotonically to a steady state level (tonic tension) and relaxes immediately upon repolarization (1). In marked contrast, voltage clamp depolarization of mature mammalian myocardium results in a biphasic response

Received November 29, 1988; accepted March 28, 1989.

Correspondence Thomas Klitzner, M.D., Ph.D., Department of Pediatrics, UCLA School of Medicine, Los Angeles, CA 90024.

Supported in part by funding from the NIH (HL01347 and HL35783), the American Heart Association, Greater Los Angeles Affiliate (829 GI), and the Variety Club, J. H. Nicholson Endowment. characterized by an early peak of tension (phasic tension) which then relaxes to a steady state level $(1,2)$. It has been postulated that the lack of phasic tension in the immature myocardium reflects structural and functional immaturity of the t-tubules and SR $(1,3)$. To further investigate this hypothesis, experiments were conducted using the alkaloid ryanodine in an attempt to selectively eliminate $\mathrm{Ca}^{2+}$ release from the SR. Ryanodine has been shown to diminish release of $\mathrm{Ca}^{2+}$ from the $\mathrm{SR}$, either by directly inhibiting $\mathrm{Ca}^{2+}$ release mechanisms (4-6), or by enhancing $\mathrm{Ca}^{2+}$ efflux, resulting in $\mathrm{Ca}^{2+}$ depletion of the $\mathrm{SR}(7,8)$, with little or no effect on the amplitude of membrane $\mathrm{Ca}^{2+}$ currents $(9,10)$ or the function of contractile proteins $(11)$. It has been demonstrated previously that the negative inotropic effect of ryanodine is less pronounced in immature than mature mammalian myocardium (12-14) suggesting that it may be a useful tool in studying the changes which occur in the mechanisms of myocardial excitation-contraction coupling with development.

\section{MATERIALS AND METHODS}

Preparation. Right ventricular papillary muscles, $0.3-0.7 \mathrm{~mm}$ in diameter, were dissected from seven newborn (0-10 days) and six adult New Zealand White rabbits. Muscles were isolated and placed in a three compartment single sucrose gap, voltage clamp chamber. The tendinous end of the papillary muscle was fastened with fine suture to an isometric tension transducer in the physiologic chamber and the cut end was pulled through adjustable holes in the rubber membranes separating the larger outer compartments from the central chamber. The cut end was left loose in the outer chamber containing KCl-Tyrode's solution $(\mathrm{KCl}$ $128 \mathrm{mM} ; \mathrm{KHCO}_{3} 24 \mathrm{mM}$ ). Typically $0.4-1.0 \mathrm{~mm}$ of the tendinous end of the muscle protruded into the physiologic compartment through which oxygenated Tyrode's solution $(\mathrm{NaCl} 118$ $\mathrm{mM} ; \mathrm{CaCl}_{2} 1.0 \mathrm{mM}$; glucose $6 \mathrm{mM} ; \mathrm{MgCl}_{2} 1.0 \mathrm{mM} ; \mathrm{KCl} 4.0$ $\mathrm{mM} ; \mathrm{NaHCO}_{3} 24 \mathrm{mM} ; \mathrm{NaH}_{2} \mathrm{PO}_{4} 0.435 \mathrm{mM}$ ) flowed at a constant rate $(2-3 \mathrm{~mL} / \mathrm{min})$. Oxygenated, isosmotic sucrose (sucrose $280 \mathrm{mM}$-high purity, grade I, Sigma Chemical Co., St. Louis, MO) flowed at a rapid rate $(2-6 \mathrm{~mL} / \mathrm{min})$ through the middle compartment. Preparations were stimulated at six shocks/min by passing 5 -ms current pulses at twice threshold current and were allowed to stabilize in the experimental chamber for 45 min before the start of the experiment. Control Tyrode's solution was equilibrated with $95 \% \mathrm{O}_{2}$ and $5 \% \mathrm{CO}_{2}$, and all experiments were conducted at $20-23^{\circ} \mathrm{C}$. Membrane potential, membrane current, and developed tension were recorded on a storage oscilloscope and photographed for later analysis. Details of the single sucrose gap voltage clamp technique including its validity, limitations, and application to neonatal papillary muscles have been discussed previously $(1,15,16)$.

Experimental protocol. Developed tension was measured in response to $2-\mathrm{s}$ voltage clamp depolarizations to $+10 \mathrm{mV}$ substi- 
tuted for one of the stimulated action potentials. Tonic tension was defined as developed tension at the end of a clamp step. Phasic tension was defined as the maximum early peak of tension seen in response to a voltage clamp step. When no clear early peak of tension could be identified, such as in neonatal myocardium (1), phasic tension was measured at the point where a line drawn tangent to the final tonic tension intersected a line drawn tangent to the rising phase of tension. This value was often less than the tonic tension measured for the same clamp step. The ratio of phasic to tonic tension $(\mathrm{P} / \mathrm{T}$ ratio) was calculated for each voltage clamp depolarization. A minimum of 12 normal action potentials were stimulated between test voltage clamps. In $\mathrm{Ca}^{2+}$-loading experiments, the same voltage clamp step (2-s duration, $+10 \mathrm{mV}$ ) was substituted for two consecutive stimuli. While continuing to stimulation at six shocks/min, the physiologic portion of the preparation was exposed to $10^{-5} \mathrm{M}$ ryanodine (LVOG Inc. Agrisystems International, Wind Gap, PA) for 25 min. Voltage clamp depolarizations were then repeated. In some experiments, the level of voltage clamp depolarization was varied, both before and after addition of ryanodine, to allow determination of the voltage dependence of developed tension.

Statistical analysis. Results were analyzed by comparing tension measurements before and after ryanodine administration using paired $t$ tests. Because of the variable wt and cross-sectional area of the physiologic portion of papillary muscles, accurate normalization of tension measurements was not possible. The use of paired $t$ tests allowed each preparation to serve as its own control. An unpaired $t$ test was used to compare the degree of change in the $\mathrm{P} / \mathrm{T}$ ratio between newborn and adult myocardium upon addition of ryanodine.

\section{RESULTS}

Effect of ryanodine on phasic component of tension. Figure 1 shows original records from a typical experiment in which a papillary muscle from an adult rabbit was exposed to a 2-s voltage clamp step to $+10 \mathrm{mV}$ before and after addition of $10^{-5}$ $M$ ryanodine. Before addition of ryanodine, the muscle developed both phasic and tonic tension. Ryanodine produced a marked reduction in the phasic component of developed tension.
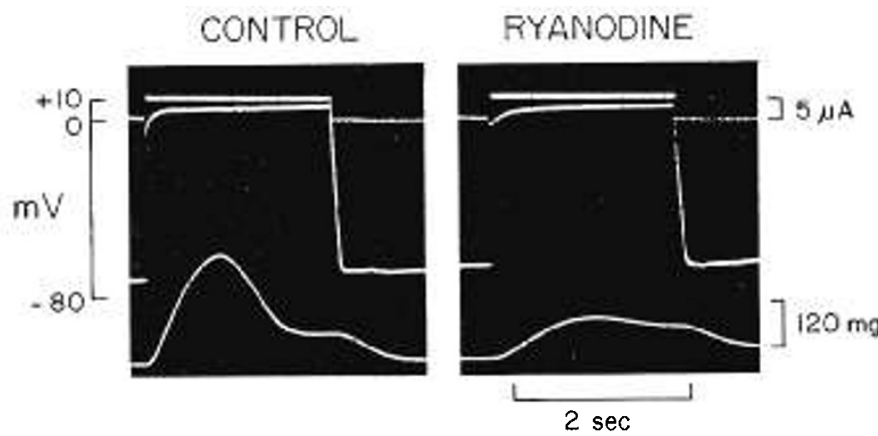

Fig. 1. Tension response of mature myocardium before and after addition of ryanodine. Two second voltage clamp depolarizations to +10 $\mathrm{mV}$ are shown for an adult papillary muscle before (left) and after (right) addition of $10^{-5} \mathrm{M}$ ryanodine. Three traces are displayed in each panel. Membrane potential is taken from rest $(-80 \mathrm{mV})$ to $+10 \mathrm{mV}$ by the voltage clamp circuitry. After a 2 -s depolarization, the membrane potential is allowed to return to rest. The current trace shown at the top of each panel represents the net membrane current which flows during the clamp step. The line at $0 \mathrm{mV}$ represents zero current flow (inward current is displayed as negative). The tension response is shown at the bottom of each panel. Note that before addition of ryanodine the mature papillary muscle responds to a voltage clamp step with both early peak (phasic) and steady state (tonic) tension. After addition of ryanodine, the muscle develops only tonic tension, the magnitude of which is unchanged by ryanodine. The postryanodine tension response is very similar to that seen in normal newborn myocardium.
As reported previously (1), the $\mathrm{P} / \mathrm{T}$ ratio is an appropriate index of the relation between $\mathrm{Ca}^{2+}$ release from the $\mathrm{SR}$ and $\mathrm{Ca}^{2+}$ influx across the sarcolemma. The $\mathrm{P} / \mathrm{T}$ ratio for the six adult papillary muscles tested decreased significantly from $2.04 \pm 0.40$ to $1.03 \pm 0.14$ (mean \pm SEM) upon exposure to ryanodine ( $p<$ $0.05)$. These findings are consistent with the hypothesis that the phasic component of voltage clamp induced tension in adult mammalian myocardium results from release of $\mathrm{Ca}^{2+}$ from the SR.

In the seven newborn papillary muscles, ryanodine caused a small but significant decrease in the $\mathrm{P} / \mathrm{T}$ ratio from $1.1 \pm 0.05$ to $0.97 \pm 0.03(p<0.05)$. This decrease was significantly less than that seen in the adult group $(p<0.05)$. These results suggest that internal storage and release of $\mathrm{Ca}^{2+}$ by the $\mathrm{SR}$ may exist to a limited extent in newborn myocardium, but are significantly less important for tension generation in this age group as compared with the adult.

We have reported previously that newborn myocardium can develop significant phasic tension when a conditioning clamp step is imposed immediately preceding the test clamp step (1). Accordingly, two clamp, $\mathrm{Ca}^{2+}$-loading experiments were conducted before and after administration of ryanodine in papillary muscles from the seven newborn rabbits. Before administration of ryanodine, $\mathrm{Ca}^{2+}$ loading resulted in a significant increase in the $\mathrm{P} / \mathrm{T}$ ratio when the initial or conditioning clamp step was compared with the subsequent test depolarization $(1.1 \pm 0.05$ to $2.07 \pm 0.27 ; p<0.05)$. After administration of ryanodine an identical $\mathrm{Ca}^{2+}$-loading protocol failed to produce an increase in the $\mathrm{P} / \mathrm{T}$ ratio $(0.97 \pm 0.03$ preryanodine and $1.01 \pm 0.11$ postryanodine; $p=\mathrm{NS}$ ). These results suggest that the augmentation of phasic tension which follows $\mathrm{Ca}^{2+}$-loading in newborn myocardium is the result of increased $\mathrm{Ca}^{2+}$ content and subsequent increased $\mathrm{Ca}^{2+}$ release from the $\mathrm{SR}$.

Effects of ryanodine on the tonic component of tension. Tonic tension, in both mammalian and amphibian ventricular tissue has been shown to be under the direct control of the membrane potential $(1,2,17)$. Thus, it has been postulated that this tension component is generated by an influx of $\mathrm{Ca}^{2+}$ across the sarcolemma $(15,17)$. It may be seen in the tension records of Figure 1 that ryanodine had a significant effect on phasic tension, but did not appear to alter tonic tension. In the six adult papillary muscles studied, tonic tension did not change significantly with the addition of ryanodine ( $104 \pm 15 \mathrm{mg}$ preryanodine and 102 $\pm 16 \mathrm{mg}$ postryanodine; $p=\mathrm{NS}$ ). Similarly, ryanodine produced no significant change in the tonic component of tension in the newborn group $(96 \pm 19 \mathrm{mg}$ preryanodine and $99 \pm 20 \mathrm{mg}$ postryanodine; $p=\mathrm{NS}$ ). These results support the notion that the action of ryanodine is limited to diminishing $\mathrm{SR} \mathrm{Ca}{ }^{2+}$ release and that sarcolemmal $\mathrm{Ca}^{2+}$ transport is not significantly affected.

Effect of ryanodine on voltage dependence of developed tension. Additional insight into mechanisms of tension generation may be obtained by determining the voltage dependence of the various components of developed tension. Voltage dependence was analyzed by varying the amplitude of voltage clamp depolarizations and measuring each tension component at different levels of membrane potential. Figure 2 shows a graphic representation of the tonic tension-voltage relation in a typical adult papillary muscle. Tonic tension began to develop between -50 and -60 $\mathrm{mV}$ and was a monotonically increasing function of membrane potentials both before and after administration of ryanodine. Similar results are shown in Figure 3 measuring tonic tension before and after ryanodine administration in a typical newborn papillary muscle.

In contrast, ryanodine had a marked effect on the phasic tension-voltage relation. Before the administration of ryanodine, the phasic tension-voltage relation of a typical adult papillary muscle (Fig. 4) was similar to that reported previously $(1,2)$. Phasic tension began to develop at approximately $-50 \mathrm{mV}$ and increased with increasing membrane potential up to approximately $0 \mathrm{mV}$ where it reversed and began to decline. After 


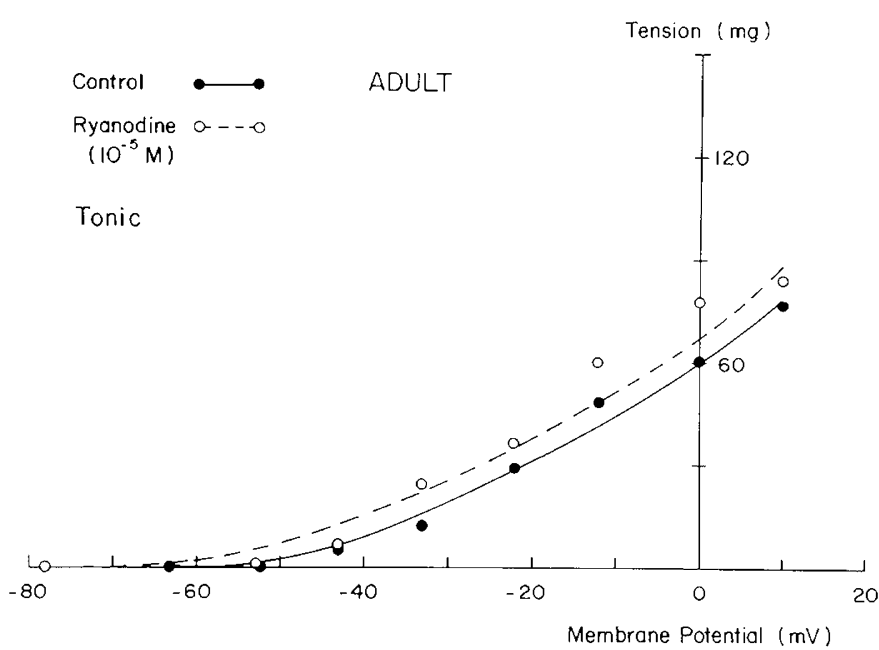

Fig. 2. Tension-voltage relations for tonic tension before and after addition of ryanodine in mature myocardium. The relationship between tonic tension and voltage is plotted for 2-s voltage clamp steps in typical adult papillary muscle before (closed circles) and after (open circles) addition of $10^{-5} \mathrm{M}$ ryanodine. Tension-voltage relations are generated by varying the clamp potential (plotted on the $\mathrm{x}$-axis) and measuring the developed tension (plotted on the y-axis). Tonic tension begins to develop at -50 to $-60 \mathrm{mV}$ and increases with increasing membrane potential up to the highest potential tested. Addition of ryanodine did not result in a significant change in the magnitude or voltage dependence of tonic tension.

administration of ryanodine, preparations often did not develop a distinct early peak of voltage clamp-induced tension (see Fig. 1). Therefore, the postryanodine "phasic" tension-voltage relation was constructed by measuring developed tension at a time comparable to the time of the peak of phasic tension before the addition of ryanodine. As demonstrated in Figure 4, the postryanodine "phasic" tension-voltage relation was a monotonically increasing function of membrane potential, similar to the tension-voltage relation for tonic tension in both the adult and newborn.

\section{DISCUSSION}

The major findings of this investigation are that ryanodine suppressed the development of phasic tension in mature myocardium, and thus abolished a major difference in tension generation between the newborn and adult. In the immature myocardium, ryanodine caused a small but significant decrease in the ratio of phasic to tonic tension. Further, the augmentation in phasic tension induced by $\mathrm{Ca}^{2+}$-loading of newborn myocardium was abolished by ryanodine. In the adult, the residual early tension component remaining after ryanodine administration had a voltage dependence comparable to that of tonic tension. In both age groups, ryanodine did not cause a change in the amplitude or voltage dependence of tonic tension.

Implications for generation of the tonic component of tension. It has been reported previously that ryanodine does not influence the amplitude of membrane $\mathrm{Ca}^{2+}$ currents $(9,10)$. There have not been reports of a significant effect of ryanodine on other mechanisms of sarcolemmal $\mathrm{Ca}^{2+}$ influx (18). The present experiments suggest that ryanodine's action on voltage clampinduced tension is limited to a diminution of the phasic tension component with no effect on tonic tension in either the newborn or adult age group (Figs. 2 and 3). Inasmuch as the development of tonic tension is most likely dependent upon $\mathrm{Ca}^{2+}$ influx $(15$, 17 ), it is probable that ryanodine does not significantly affect any of the sarcolemmal transport mechanisms responsible for delivering activator $\mathrm{Ca}^{2+}$ to the myofilaments. Nonetheless, another

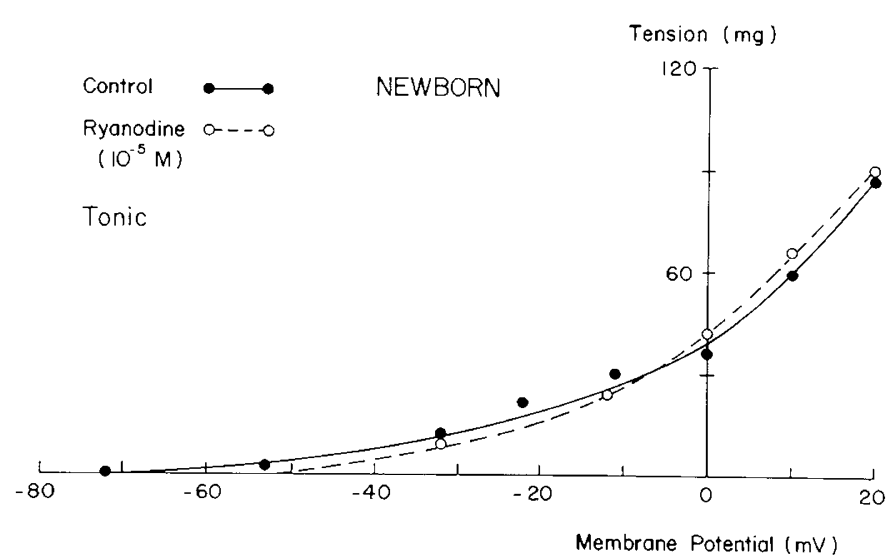

Fig. 3. Tension-voltage relations for tonic tension before and after addition of ryanodine in newborn myocardium. The relationship between tonic tension and voltage is plotted for 2-s voltage clamp steps in a typical newborn papillary muscle before (closed circles) and after (open circles) addition of $10^{-5} \mathrm{M}$ ryanodine. Similar to the adult, tonic tension begins to develop at -50 to $-60 \mathrm{mV}$ and increases with increasing membrane potential up to the highest potential tested. As in the adult, addition of ryanodine produced no significant change in the magnitude or voltage dependence of tonic tension.

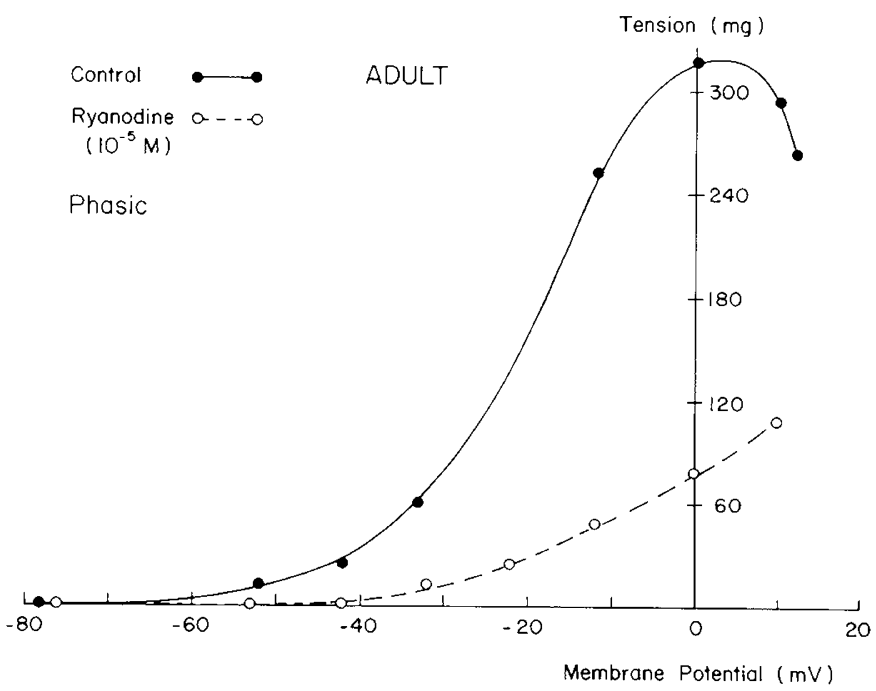

Fig. 4. Tension-voltage relations for phasic tension before and after addition of ryanodine in adult myocardium. The relationship between phasic tension and voltage is plotted for 2 -s voltage clamp steps in a typical adult papillary muscle before (closed circles) and after (open circles) addition of ryanodine. Phasic tension begins to develop at -50 to $-60 \mathrm{mV}$ and increases with increasing membrane potential up to 0 $\mathrm{mV}$ where it begins to decline with increasing membrane potential. Addition of ryanodine significantly reduced the amplitude of phasic tension and altered its voltage dependence to resemble tonic tension. This change in voltage dependence suggests that after addition or ryanodine, early tension is no longer the result of $\mathrm{Ca}^{2+}$ release from the sarcoplasmic reticulum, but may reflect early $\mathrm{Ca}^{2+}$ entry from the extracellular space.

possible explanation is that ryanodine does depress inward $\mathrm{Ca}^{2+}$ flux, while simultaneously decreasing $\mathrm{Ca}^{2+}$ uptake by a similar amount. Evidence against this possibility is provided by studies of isolated SR which suggest that ryanodine does not depress $\mathrm{Ca}^{2+}$ uptake (4). Therefore, it is likely that ryanodine acts as a selective inhibitor of SR-dependent tension generation as proposed by Sutko et al. (18), and may be a useful pharmacologic 
probe to differentiate this mechanism of tension generation from one that is dependent on sarcolemmal $\mathrm{Ca}^{2+}$ influx.

Implications for generation of phasic component of tension. It has been postulated previously that the phasic component of voltage clamp controlled tension in mammalian myocardium is produced by release of $\mathrm{Ca}^{2+}$ from the SR $(1,2)$. The results of this investigation are compatible with this hypothesis. Ryanodine inhibited phasic tension in adult myocardium (Fig. 1) and appears to have altered the mechanism of tension generation in this age group to closely resemble that of the normal newborn, i.e. dependent on sarcolemmal $\mathrm{Ca}^{2+}$ influx.

The finding that ryanodine produced a small but significant decrease in the $\mathrm{P} / \mathrm{T}$ ratio of newborn msucles suggests that under the experimental conditions investigated here, phasic tension does exist in newborn myocardium but is significantly reduced compared to that seen in the adult. In addition, ryanodine's abolition of phasic tension induced by $\mathrm{Ca}^{2+}$ loading suggests that conditioning clamp protocols augment phasic tension by increasing the $\mathrm{Ca}^{2+}$ content of the SR. Consequently, it may be the case that the mechanism for release of $\mathrm{Ca}^{2+}$ from the SR is present and functional in the newborn period, but participation of the $\mathrm{SR}$ in tension development is reduced because of a diminished $\mathrm{SR} \mathrm{Ca}^{2+}$ content. This speculation is consistent with the decreased ability to accumulate $\mathrm{Ca}^{2+}$ demonstrated in vesicles of SR isolated from immature lamb, guinea pig, and rabbit (1921 ). However, it should be recognized that the $\mathrm{Ca}^{2+}$ content of the SR may be higher at physiologic heart rates and temperatures than under the experimental conditions used in these studies and those of other investigators.

Finally, an alternative explanation is suggested in regard to the proposal of Nassar et al. that the lack of diminution of an extrasystolic beat in ventricular myocytes isolated from 3-wk-old rabbits indicates proximity of $\mathrm{Ca}^{2+}$ uptake and release sites in the immature SR (22). Inasmuch as the immature myocardium is largely dependent on sarcolemmal $\mathrm{Ca}^{2+}$ influx for tension generation, $\mathrm{Ca}^{2+}$ from the SR would not be expected to contribute significantly to either the control or extrasystolic beat. However, the $\mathrm{Ca}^{2+}$ load delivered by the extrasystolic beat might raise $\mathrm{SR} \mathrm{Ca}^{2+}$ content resulting in postextrasystolic potentiation, especially at $3 \mathrm{wk}$ of age when, as we have shown previously (1), the SR is capable of significant $\mathrm{Ca}^{2+}$ sequestration and rerelease.

In conclusion, our results confirm previous investigations suggesting that the role of the SR is significantly reduced in the newborn period when compared with mature myocardium (1$3,13,23)$. The $\mathrm{Ca}^{2+}$-loading experiments suggest that the reduced participation of the SR in tension development in the newborn period may be secondary to reduced $\mathrm{Ca}^{2+}$ content of the SR rather than structural immaturity or decreased ability to release stored $\mathrm{Ca}^{2+}$.

\section{REFERENCES}

1. Klitzner T, Friedman WF 1988 Excitation-contraction coupling in developing mammalian myocardium: evidence from voltage clamp studies. Pediatr Res 23:428-432

2. Morad M, Goldman Y 1973 Excitation-contraction coupling in heart muscle: membrane control of development of tension. Prog Biophysics Mol Biol 27:257-313

3. Maylie JG 1982 Excitation-contraction coupling in neonatal and adult myocardium of cat. Am J Physiol 242:H834-H843

4. Jones LR, Besch HR Jr, Sutko JL, Willerson JT 1979 Ryanodine-induced stimulation of net $\mathrm{Ca}^{++}$uptake by cardiac sarcoplasmic reticulum vesicles. $\mathbf{J}$ Pharmacol Exp Ther 209:48-55

5. Sutko JL, Kenyon JL, 1983 Ryanodine modification of cardiac muscle responses to potassium-free solutions: evidence for inhibition of sarcoplasmic reticulum calcium release. J Gen Physiol 82:385-404

6. Feher JJ, Lipford GB, 1985 Mechanism of action of ryanodine on cardiac sarcoplasmic reticulum. Biochemica et Biophysica Acta 813:77-86

7. Meissner $\mathrm{G} 1986$ Ryanodine activation and inhibition of the $\mathrm{Ca}^{2+}$ release channel of sarcoplasmic reticulum. J Biol Chem 261:6300-6306

8. Bers DM, Bridge JH, MacLeod KT 1987 The mechanism of ryanodine action in rabbit ventricular muscle evaluated with $\mathrm{Ca}$-selective microelectrodes and rapid cooling contractures. Can J Physiol Pharmacol 65:610-618

9. Mitchell MR, Powell T, Terrar DA, Twist VW 1984 Ryanodine prolongs Cacurrents while suppression contraction in rat ventricular muscle cells. $\mathrm{Br} \mathrm{J}$ Pharmacol 81:13-15

10. Marban E Wier WG 1985 Ryanodine as a tool to determine the contributions of calcium entry and calcium release to the calcium transient and contraction of cardiac purkinje fibers. Circ Res 56:133-138

11. Nayler WG, Daile P, Chipperfield D, Gan K 1970 Effect of ryanodine on calcium in cardiac muscle. Am J Physiol 219:1620-1626

12. Penefsky ZJ 1974 Studies on mechanism of inhibition of cardiac muscle contractile tension by ryanodine. Mechanical response. Pflugers Arch 347:173-184

13. Nakanishi T, Okuda H, Kamata K, Abe K, Sekiguchi M, Takao A 1987 Development of myocardial contractile system in the fetal rabbit. Pediatr Res 22:201-207

14. Seguchi M Harding JA, Jarmakani JM 1986 Developmental change in the function of sarcoplasmic reticulum. J Mol Cell Cardiol 18:189-195

15. Morad M, Orkand RK 1971 Excitation-contraction coupling in frog ventricle: evidence from voltage clamp studies. J Physiol 219:167-189

16. Goldman Y, Morad M 1977 Measurement of transmembrane potential and current in cardiac muscle: a new voltage clamp method. J Physiol 268:613654

17. Klitzner T, Morad M 1983 Excitation-contraction coupling in frog ventricle: possible $\mathrm{Ca}^{2+}$ transport mechanisms. Pflugers Arch 398:274-283

18. Sutko JL, Katsuaki I, Kenyon J 1985. Ryanodine: a modifier of sarcoplasmic reticulum calcium release in striated muscle. Fed Proc 44:2984-2988

19. Sheldon CA, Friedman WF, Sybers HD 1976 Scanning electron microscopy of fetal and neonatal lamb cardiac cells. J Mol Cell Cardiol 8:853-862

20. Mahoney L 1988 Maturation of calcium transport in cardiac sarcoplasmic reticulum. Pediatr Res 24:639-643

21. Nayler WG, Fassold E 1977 Calcium accumulating and ATPase activity of cardiac sarcoplasmic reticulum before and after birth. Cardiovasc Res 11:231-237

22. Nassar R, Reedy MC, Anderson PAW 1987 Developmental changes in the ultrastructure and sarcomere shortening of the isolated rabbit ventricular myocyte. Circ Res 61:465-483

23. Jarmakani JM, Nakanishi T, George BL, Bers DM 1982 The effect of extracellular calcium on myocardial mechanical function in the neonatal rabbit Dev Pharmacol Ther 5:1-13 